\title{
High resolution structure of the membrane embedded skeletal
}

\section{muscle ryanodine receptor}

\author{
Zephan Melville ${ }^{1}$, Kookjoo Kim ${ }^{1}$, Oliver B. Clarke ${ }^{1,2}$, Andrew R. Marks ${ }^{1,3}$ \\ ${ }^{1}$ Department of Physiology and Cellular Biophysics, ${ }^{2}$ Department of Anesthesiology, ${ }^{3}$ Clyde \& Helen Wu \\ Center for Molecular Cardiology, Columbia University Vagelos College of Physicians \& Surgeons
}

Address correspondence to: arm42@cumc.columbia.edu 


\begin{abstract}
The type 1 ryanodine receptor (RyR1)/calcium release channel on the sarcoplasmic reticulum (SR) is required for skeletal muscle excitation-contraction coupling and is the largest known ion channel, comprised of four $565 \mathrm{kDa}$ protomers. Cryogenic electron microscopy (cryoEM) studies of the RyR have primarily used detergent to solubilize the channel, though a recent study resolved the structure with limited resolution in nanodiscs ${ }^{1}$. In the present study we have used cryoEM to solve high-resolution structures of the channel in liposomes using a gel-filtration approach with on-column detergent removal to form liposomes and incorporate the channel simultaneously, a method that improved the incorporation rate by more than 20-fold compared to a dialysis-based approach. In conjunction with new direct-detection cameras, this allowed us to resolve the structure of the channel in the closed and open states at 3.36 and $3.98 \AA$, respectively. This method offers validation for detergent-based structures of the RyR and lays the groundwork for studies utilizing an electrochemical gradient mimicking the native environment, such as that of the SR, where $\mathrm{Ca}^{2+}$ concentrations are millimolar in the lumen and nanomolar in the cytosol of the cell at rest.
\end{abstract}




\section{Introduction}

Located on the sarco/endoplasmic reticulum (SR/ER) membrane, the two megadalton ryanodine receptor $(\mathrm{RyR})$ is the largest known ion channel ${ }^{2,3}$. In skeletal muscle, RyR1 is required for $\mathrm{SR} \mathrm{Ca}^{2+}$ release during excitation-contraction coupling (EC coupling). There are three RyR isoforms, RyR1 is the primary isoform in skeletal muscle while RyR2 is the cardiac isoform, though both are found in numerous tissues including neurons $s^{4-6}$. RyR3 is widely expressed but at significantly lower levels ${ }^{7}$. In skeletal muscle, RyR1 is thought to be activated by direct interaction with the dihydropyridine receptor (DHPR $)^{8,9}$, whereas RyR2 is activated by $\mathrm{Ca}^{2+}$ in a process termed calcium-incuded calcium release $(\mathrm{CICR})^{10,11}$. In short, $\mathrm{Ca}^{2+}$ influx via the DHPR triggers the release of $\mathrm{Ca}^{2+}$ via RyR2, which in turn creates a high local concentration of $\mathrm{Ca}^{2+}$ that activates neighboring RyR2 tetramers. RyR channels are tightly packed in checkerboard arrays on the SR and exhibit cooperative activation/deactivation through the process termed coupled gating ${ }^{12-15}$. Leaky RyR channels are associated with numerous diseases and disorders including muscular dystrophy ${ }^{16}$, heart failure ${ }^{17}$, cardiac $\operatorname{arrhythmias}^{18}$, diabetes $^{19}$, Huntington's Disease ${ }^{20-22}$, and Alzheimer's ${ }^{2,3,23,24}$.

The high resolution structures of individual, detergent solubilized RyR $1^{25-28}$ and RyR $2^{29}$ channels have been solved using cryogenic electron microscopy $(\mathrm{cryoEM})^{15}$; however, solving the structure of proteins in native or near-native membranes remains challenging. Most cryoEM structures of membrane proteins use detergent micelles, amphipols, or nanodiscs ${ }^{30-34}$. Liposomes, along with linear and circularized nanodiscs, allow for membrane protein incorporation while polymers such as DIBMA and SMA allow for native membrane extractions into so called native nanodiscs $35-38$; however, critically, liposomes are the only method that allows for the creation of asymmetric environments, such as that of the SR. In the lumen of the SR, millimolar concentrations of $\mathrm{Ca}^{2+}$ are stored and maintained by the sarco/endoplasmic reticulum $\mathrm{Ca}^{2+}$ ATPase (SERCA) and calsequestrin, while the cytosol of the cell remains at approximately $150 \mathrm{nM} \mathrm{Ca}^{2+}$ at rest and low micromolar following $\mathrm{Ca}^{2+}$ release through the RyR. Liposomes form spontaneously as detergent is removed from solution during dialysis, or through the use of Bio-beads that adsorb detergent molecules. Lige Tonggu \& Liguo Wang have shown that liposomes can also be formed via gel filtration ${ }^{39}$. This method allows for significantly greater control over the size of the liposomes formed compared to 
dialysis $^{1}$ and allowed for the successful incorporation of the large conductance calcium-activated potassium (BK) channel into liposomes ${ }^{40}$.

Given the critical role of the RyR in normal and pathologic physiology, it is important to understand the structure and dynamics of the channel in the native context and to compare these structures to detergent solubilized channels. To this end, the structure and dynamics of proteoliposomal RyR1 was found to match that of detergent, offering validation for the detergent-resolved structures. As with detergent-resolved structures of the RyR, the dynamics of the cytosolic shell undergoes dramatic conformation changes in both the open and closed states of RyR1 channels. These movements are separate from the opening of the channel, marked by the dilation of the pore; however, these changes are difficult to understand in the context of an array of channels, each of which is connected to its neighbors at the four corners of the cytosolic domain ${ }^{41-}$ ${ }^{43}$. In a truly native context, these dynamics should be muted, but it seems a fully-native approach may be necessary to capture these coupled channels. While we were unable to observe any such coupled channels in liposomes, we have successfully resolved the structure of RyR1 in liposomes in both the closed and open states to 3.36 and $3.98 \AA$, respectively, through a simple adaption of the gel filtration approach of liposome formation and incorporation. In doing so, we have established a framework for studies more closely mimicking the native environment.

\section{Results}

Our approach, detailed in Methods, is based on the method of Tonggu \& Wang ${ }^{39}$ and resulted in small, consistent liposomes compared to forming liposomes by dialysis, which resulted in a wide range of liposome sizes (Figure 1A). Critically, this method also offered a greater than 20-fold increase in the RyR1 incorporation rate based on the average number of particles per micrograph with approximately 8 channels per micrograph compared to 1 particle per 3 micrographs observed in the dialysis-based approach. Unfortunately, while both methods of liposome formation required an additional clearing step, liposomes formed via gel filtration showed only minor improvement from passage over an ion-exchange column. While many particles of unincorporated RyR1 were still present, these were discarded during $2 \mathrm{~d}$ 
classification by increasing the box size to ensure the membrane would be clear and present as well as increasing the number of classes to weed out unincorporated RyR1. Despite the lack of top and bottom views of the channel, $360^{\circ}$ of side views were present in addition to oblique views (Figure S2), allowing for a full $3 \mathrm{~d}$ reconstruction of the channel in a lipid membrane, showing the potential of this method to achieve high-resolution cryoEM structures of proteins in membranes.

The structure of proteoliposomal RyR1 matches the detergent-resolved structure ${ }^{26}$ with the exception of the hydrophobic-gate residue, I4937. In liposomes, this pore helix rotates such that I4937 is no longer within the pore in the open state of the channel. As a result, the radius of the pore residue cannot not be measured at I4937 (Figure 2) (4) $^{4}$ though by estimation it remains at approximately $4 \AA$, which is consistent with the 4 $\AA ̊$ radius or $8 \AA$ diameter observed in detergent. The lumen of RyR 1 at the base of the channel varies between liposomes and detergent but this region consists of unstructured loops so dynamic behavior is to be expected. Analysis of the calstabin binding site, along with the binding sites for calcium, ATP, and caffeine show no significant changes between detergent solubilized and liposome embedded RyR1 channels for both the closed and open states, however the improved resolution in the closed state allows for unambiguous assignment of the orientation of caffeine within the binding site (Figure 1C).

The juxtamembrane helices at the top of the TM interface in each protomer make several contacts with the lipid membrane, however the lipid membrane is too dynamic to clearly identify these interactions. These helices are likely responsible for the flattening of the lipid membrane around the TM domain of the RyR that was consistently present in 2D classes (Figure 1B). Likewise, the dynamics of the cytosolic shell of the liposome embedded channel conform to what has previously been observed. The detergent solubilized RyR1 structure undergoes dramatic conformation changes that are separate from channel opening, in both

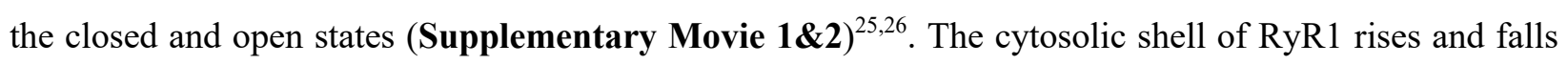
analogous to flapping wings. This movement is matched in the liposome embedded channels (Supplementary Movie 3\&4), though these movements are likely to be exaggerated in the absence of an array of channels. 


\section{Discussion}

The present study provides refined methodology that can be applied to other membrane proteins including receptors and channels. Additional cleanup steps may be required to eliminate empty liposomes and denatured protein. Since functional studies of RyR1 are often performed using planar lipid bilayers ${ }^{45}$, solving the structure in a similar membrane establishes that these channels are likely in physiologically relevant conformations. The ability to study the structure of the channel in proteoliposomes now provides the opportunity to resolve structures of membrane proteins under markedly more physiological conditions. In the case of RyR1, the $\mathrm{Ca}^{2+}$ concentration in the lumen of the $\mathrm{SR}$ is millimolar, but the cytosolic shell is exposed to nanomolar $\mathrm{Ca}^{2+}$ at rest. $\mathrm{Ca}^{2+}$ within the SR is not simply free $\mathrm{Ca}^{2+}$, but is significantly bound to $\mathrm{Ca}^{2+}$ binding proteins including calsequestrin and going forward it may be possible to incorporate calsequestrin into the lumen of proteoliposomes for structural studies.

One limitation of the present study is that the structure of liposome embedded RyR1 channels remains limited to single RyR1 channels, while native RyR1 exists in an array of channels in a checkerboard pattern on the terminal cisternae of the SR membrane $e^{41-43}$. Although as many as three molecules of RyR1 were sometimes present in a single liposome, no intermolecular interactions were observed ${ }^{46-48}$. Curiously, multiple channels were only observed in small liposomes ( $<100 \mathrm{~nm}$ diameter); however, no more than a single molecule of RyR1 was observed in liposomes $>100 \mathrm{~nm}$. Instead, larger liposomes were more likely to be empty. Thus, a structure of RyR1 in native SR membranes will be necessary to observe RyR1 arrays and the native intermolecular dynamics. In addition to validation of existing structures of detergent solubilized RyR1, the present study provides a road map for studies of RyR1 structures in native membranes at high resolution using $\mathrm{cryoEM}^{49}$, including studies mimicking the electrochemical gradient between the cytoplasm of the cell and the lumen of the SR.

\section{Methods}

The workflow for solving the high-resolution structure of RyR1 embedded in liposomes is outlined in Figure S1. 


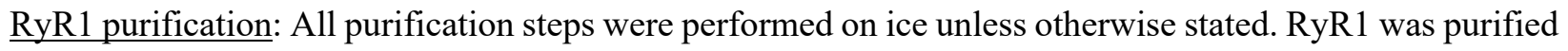
from rabbit skeletal muscle as described previously with modifications ${ }^{50}$. Rabbit back and thigh skeletal muscle, purchased from BioIVT, was snap frozen in liquid nitrogen immediately following euthanasia prior to storage at $-80^{\circ} \mathrm{C} .20 \mathrm{~g}$ of rabbit skeletal muscle was homogenized in a Waring blender and lysed in buffer A $[10 \mathrm{mM}$ tris maleate $\mathrm{pH}$ 6.8, $1 \mathrm{mM}$ EGTA, $1 \mathrm{mM}$ benzamidine hydrochloride, $0.5 \mathrm{mM}$ 4-(2aminoethyl)benzenesulfonyl fluoride hydrochloride (AEBSF)]. The resulting suspension was pelleted by centrifugation for ten minutes at $11,000 \mathrm{Xg}$. The supernatant was filtered through cheesecloth to remove debris and the membranes were then pelleted by centrifugation for thirty minutes at $36,000 \mathrm{X} \mathrm{g}$. The membranes were then solubilized in buffer B [10 mM HEPES pH 7.4, $0.8 \mathrm{M} \mathrm{NaCl}, 1 \%$ CHAPS, $0.1 \%$ phosphatidylcholine, $1 \mathrm{mM}$ EGTA, $2 \mathrm{mM}$ DTT, $0.5 \mathrm{mM}$ AEBSF, $1 \mathrm{mM}$ benzamidine hydrochloride, 1 protease inhibitor tablet (Pierce)] prior to homogenization using a glass tissue grinder (Kontes). Homogenization was repeated following the addition of buffer $\mathrm{C}$ (buffer $\mathrm{B}$ with no $\mathrm{NaCl}$ ) at a 1:1 mixture with buffer B. The resulting homogenate was subjected to centrifugation for thirty minutes at $100 \mathrm{k} \mathrm{X} \mathrm{g}$. The supernatant was then vacuum filtered and loaded at $1 \mathrm{~mL} / \mathrm{min}$ onto a HiTrap Q HP column (5 mL, GE Healthcare Life Sciences) pre-equilibrated with buffer D [10 mM HEPES pH 7.4, $400 \mathrm{mM} \mathrm{NaCl,} \mathrm{1.0 \%}$ CHAPS, $1 \mathrm{mM}$ EGTA, $0.5 \mathrm{mM}$ tris(2-carboxyethyl)phosphine (TCEP), $0.5 \mathrm{mM}$ AEBSF, $1 \mathrm{mM}$ benzamidine hydrochloride, 0.01\% 1,2-dioleoyl-sn-glycero-3-phosphocholine (DOPC, Avanti)]. DOPC (25 mg/mL), dissolved in chloroform, was evaporated under nitrogen gas and resuspended in buffer D. Contaminating proteins were washed away with six column volumes $(\mathrm{CV})$ of buffer D prior to elution of RyR1 with a linear gradient from 480 to $550 \mathrm{mM} \mathrm{NaCl}$ using buffers D and E (buffer D with $600 \mathrm{mM}$ $\mathrm{NaCl})$.

Liposome formation using dialysis and initial screening: Purified RyR1 $(1 \mathrm{mg} / \mathrm{mL})$ was incubated at a ratio of 1:2,000 with a 5:3 ratio of phosphatidylethanolamine and phosphatidylcholine and dialyzed into buffer F [10 mM HEPES pH 7.4, $400 \mathrm{mM} \mathrm{NaCl}, 1 \mathrm{mM}$ EGTA, $0.5 \mathrm{mM}$ TCEP] for 48 hours replacing the buffer with fresh buffer twice per day. Initial screening was performed on a Tecnai F20 (FEI) microscope at 
Columbia University. Liposomes formed by dialysis were found to have widely variable sizes and even with passage through an extruder, many liposomes were not unilammelar.

Liposome formation using gel filtration: Purified RyR1 was concentrated to $<0.5 \mathrm{~mL}$ and incubated with 1:1,000 5:3 phosphatidylethanolamine and phosphatidylcholine in 10\% CHAPS for 10 minutes prior to loading onto a hand-packed G50 column pre-equilibrated with buffer F. Liposome formation and RyR1 incorporation occurred on the column and proteoliposomal RyR1 was eluted at $0.20-0.25 \mathrm{~mL} / \mathrm{min}$ in buffer F.

Cleanup: Proteoliposomal RyR1 was cleared via passage over a second HiTrap Q column, washed at 425 $\mathrm{mM} \mathrm{NaCl}$ then eluted at $600 \mathrm{mM} \mathrm{NaCl}$. Proteoliposomal RyR1 was concentrated to $2.5 \mathrm{mg} / \mathrm{mL}$, determined by spectroscopy using a NanoDrop 1000 (ThermoFisher, 1 abs @ 280 nm=1 mg/mL).

Grid preparation: UltrAuFoil holey gold grids (Quantifoil R 0.6/1.0, Au 300) were plasma cleaned for thirty seconds with $\mathrm{H}_{2}$ and $\mathrm{O}_{2}$ (Gatan). Prior to setting grids, proteoliposomal RyR1 was incubated for 10 min with $2 \mathrm{mM}$ NaATP, $5 \mathrm{mM}$ caffeine, and $30 \mu \mathrm{M} \mathrm{Ca}^{2+}$ free (MaxChelator) $^{51}$. These conditions were chosen to ensure both the closed and open states would be visible. $3.0 \mu \mathrm{L}$ of $2.5 \mathrm{mg} / \mathrm{mL}$ proteoliposomal RyR1 was applied to each grid. Grids were then blotted for $7.5 \mathrm{sec}$ at blot force 3, with a wait time of thirty seconds prior to vitrification by plunge freezing into liquid ethane chilled with liquid nitrogen ${ }^{52,53}$ with a Vitrobot Mark IV (ThermoFisher) operated at $4^{\circ} \mathrm{C}$ with $100 \%$ relative humidity.

Data Collection: Grids prepared with liposomes formed by gel filtration were screened at City University of New York (CUNY) using a 120-kV G2 Spirit Twin microscope (FEI Tecnai). Microscope operations and data collection were carried out using the SerialEM software ${ }^{54}$. High resolution data collection was performed at Columbia University on a Titan Krios 300-kV (ThermoFisher) microscope equipped with an energy filter (slit width $20 \mathrm{eV}$ ) and a K3 direct electron detector (Gatan). Data were collected using Leginon $^{55}$ and at a nominal magnification of $105,000 \mathrm{X}$ in electron counting mode, corresponding to a calibrated pixel size of $0.828 \AA$. The electron dose rate was set to $16 \mathrm{e}^{-/} / \mathrm{pixel} / \mathrm{sec}$ with $2.5 \mathrm{~second}$ exposures, for a total dose of $58.34 \mathrm{e} / \mathrm{A}^{2}$. These grids showed small, consistent liposomes, similar to those reported by 
by Tonggu \& Wang ${ }^{39}$ and significantly greater incorporation rate of RyR1; however, empty liposomes and unincorporated and aggregated RyR1 also remained.

Data Processing: CryoEM data processing was performed using cryoSPARC ${ }^{56-60}$ with the exception of 3D classes, which were performed in Relion 3.1 to ensure accurate comparisons of the dynamics between liposome embedded channels and the previously published detergent-resolved channels ${ }^{26,61}$. Image stacks were aligned using Patch motion correction and defocus value estimation by Patch CTF estimation. Initial particle picking was performed manually for $>100$ particles to create templates prior to template-based picking. This involved increasing the box size to ensure a portion of the lipid membrane would be included. Two million particles were initially picked from 11,187 micrographs and these were subjected to several iterations of 2D classification in cryoSPARC with 200 classes each to separate proteoliposomal RyR1 from empty liposomes and free RyR1. Classes comprised of 175,000 particles of unincorporated RyR1 were eliminated during iterative rounds of 2D classification, leaving 85,000 liposome-embedded particles. This represents a greater than 20-fold increase in RyR1 incorporation rate over the dialysis-based method which resulted in 2,600 particles from over 7,000 micrographs. The particles from the highest-resolution classes were pooled for ab initio 3D reconstruction with a single class followed by homogenous refinement with per-particle defocus refinement and C4 symmetry imposed. 3D variability analysis revealed the presence of the open state and heterogenous refinement was performed in order to separate the two states, with $63 \%$ of particles $(53,882)$ in the closed state and $37 \%$ in the open state $(31,599)$.

Symmetry expansion and local refinement, were performed using cryoSPARC to improve local resolution. Local refinement was performed using three separate masks. The first mask was composed of the Nterminal domain, the SPRY domains, the RY1\&2 domain, and calstabin. The second mask surrounded the bridging solenoid, and the third mask surrounded the RyR1 pore. The resulting maps were combined in Chimera $^{62}$ to generate a composite map prior to calibration of the pixel size using the crystal structure of the N-terminal domain $(2 \mathrm{XOA})^{63}$. Model building was performed in $\operatorname{Coot}^{64}$ with refinement in Phenix ${ }^{65,66}$ and figures were generated using Chimera ${ }^{62}$, ChimeraX ${ }^{67}$, and $\mathrm{PyMol}^{68}$. The pore aperture of RyR1 was calculated using $\mathrm{HOLE}^{44}$. Movies of the dynamics of the channel were made in ChimeraX using maps 
generated by 3D classes for liposomes and the corresponding maps from 3D Classes in detergent (5TAN,

5TAM, 5TA3, 5T9V). Each map was filtered to $5 \AA$ and aligned at the pore. CryoEM statistics are summarized in Figure S2 and Table $\mathbf{S 1 .}$

\section{Acknowledgements}

These studies were supported by NIH grants R01HL145473, R01DK118240, R01HL142903, R01HL140934, R01AR070194 and T32 HL120826 (to A.R.M.).

\section{Author contributions}

Z.M. conducted experiments, analyzed data, and wrote the manuscript; K.K. conducted experiments and analyzed data; A.R.M and O.B.C. analyzed data and edited the manuscript.

\section{Competing interests}

A.R.M. is a consultant for ARMGO Pharma and A.R.M. and Columbia University own shares in ARMGO Pharma, a biotechnology company developing ryanodine receptor-targeted drugs.

\section{Figure Legends}

Figure 1. Workflow diagram for the preparation of proteoliposomal RyR1. A. RyR1 was purified from rabbit skeletal muscle using ion-exchange chromatography prior to liposome formation and incorporation by dialysis or gel filtration. For both methods, a second round of ion-exchange chromatography was employed to eliminate unincorporated RyR1 and empty liposomes. An embedded channel is highlighted in representative micrographs. B. 2D classes of proteoliposomal RyR1 showing the flattening of the lipid membrane around the TM domain of the channel. $\boldsymbol{C}$. Representative density for the $\mathrm{Ca}^{2+}, \mathrm{ATP}$, and caffeine binding sites of RyR1 in the closed state of proteoliposomal RyR1, resolved to $3.36 \AA$. 
Figure 2. Accessible inner surface of the RyR1 pore. The transmembrane pore (residues 4820-5037) is depicted as a ribbon diagram of two protomers with the hydrophobic gate residue, I4937, in magenta. The dotted representation of the accessible inner surface of the channel was calculated using HOLE and is colored green where the radius exceeds $4 \AA$ and red when the radius is less than $4 \AA$. The transmembrane domain of RyR1 is highlighted in tan. A. Side and cytoplasmic views of the inner surface of the pore of proteoliposomal RyR1 in the closed and open states. B. Side and cytoplasmic views for the RyR1 in detergent in the closed (5taq) and open (5tal) state. C. Graphical representation of the pore radius of the channel in liposomes (black) and detergent (gray) in the closed and open states. The channel coordinates follow from the lumen to the cytosolic side of the RyR1 with the liposomal structure on the primary $\mathrm{x}$-axis and the detergent structures on the secondary $\mathrm{x}$-axis.

Figure S1. Data processing workflow for proteoliposomal RyR1 in cryoSPARC and Relion.

Figure S2. $A$. GSFSC curves of proteoliposomal RyR1 in the closed (left) and open (right) states. $B$. Viewing angle distributions of proteoliposomal RyR1. 
bioRxiv preprint doi: https://doi.org/10.1101/2021.03.09.434632; this version posted March 9, 2021. The copyright holder for this preprint (which was not certified by peer review) is the author/funder. All rights reserved. No reuse allowed without permission.

\section{Figure 1}

A
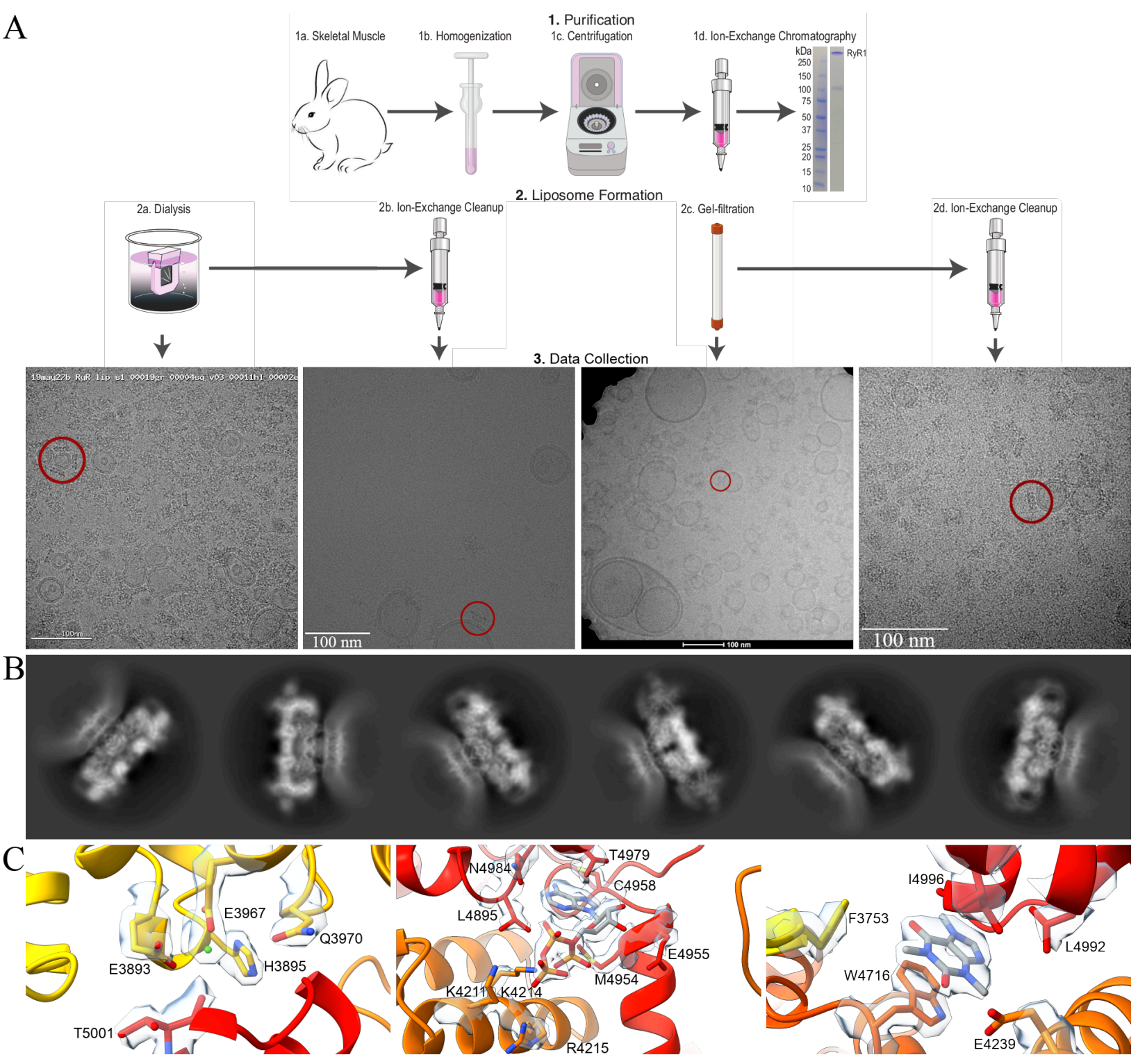
Figure 2

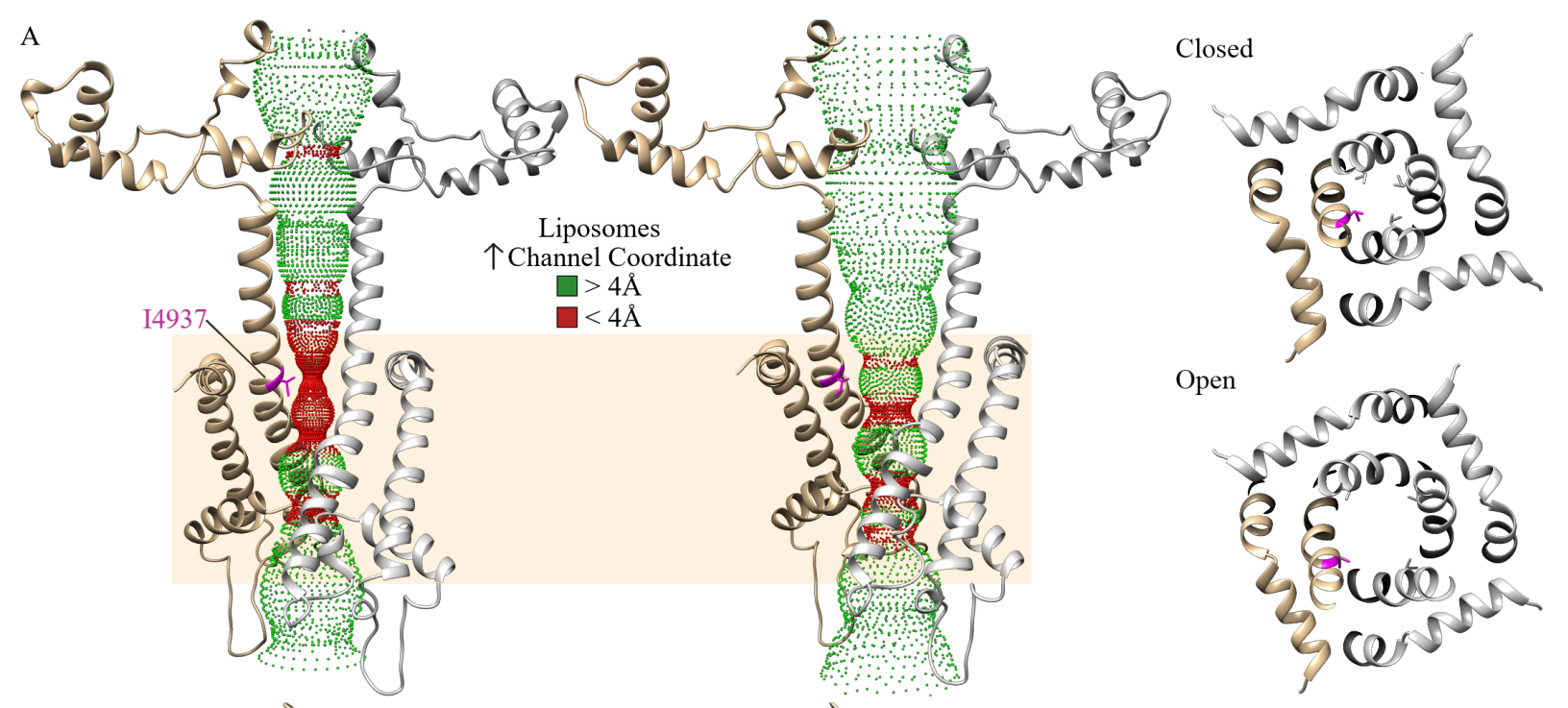

B

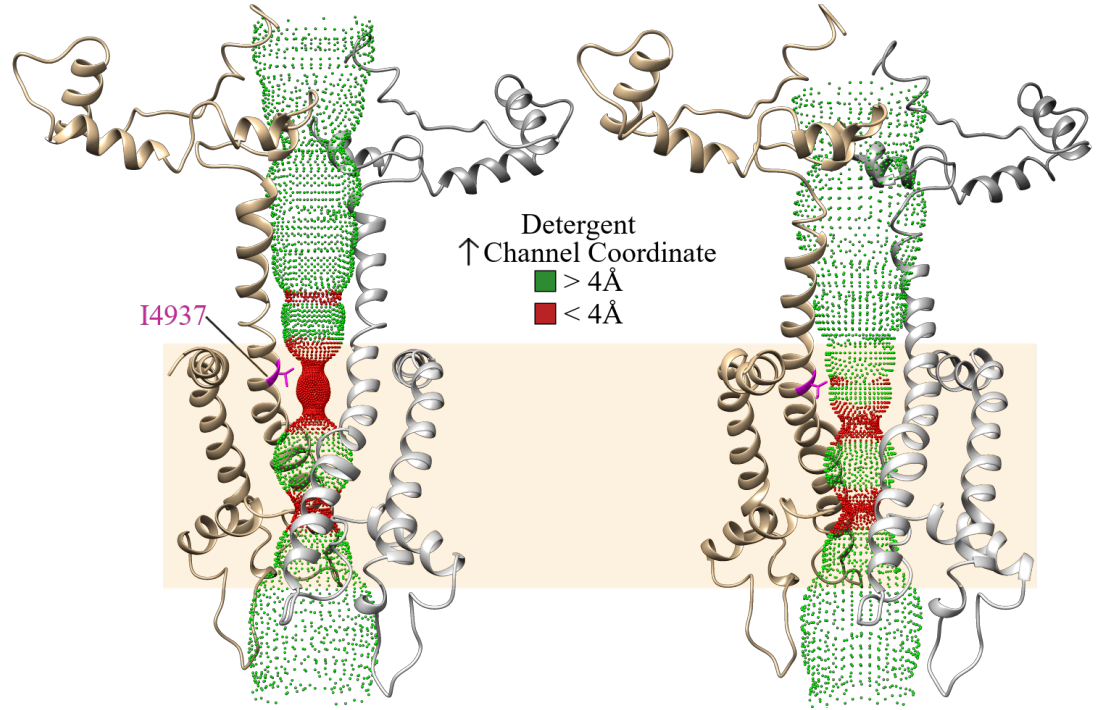

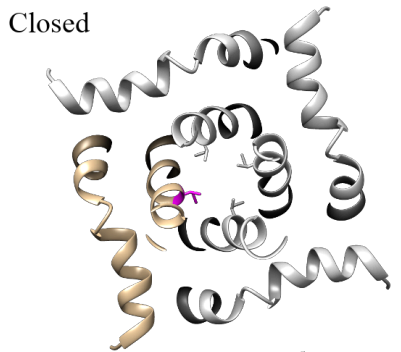

Open

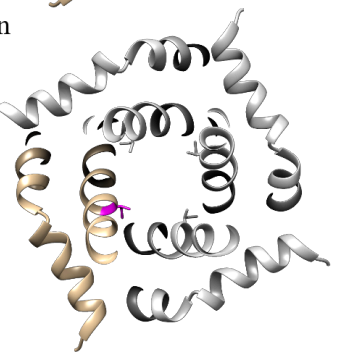

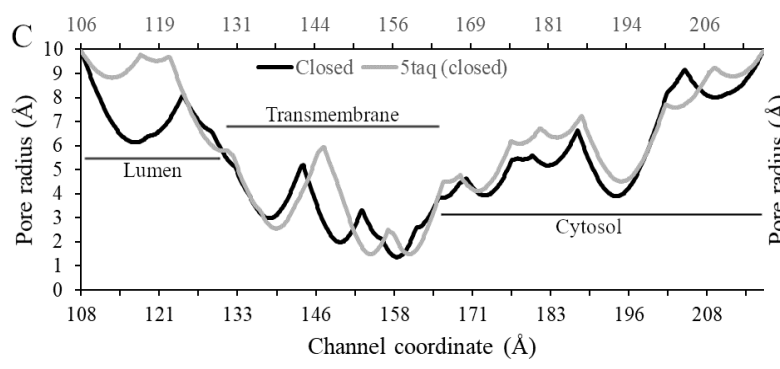

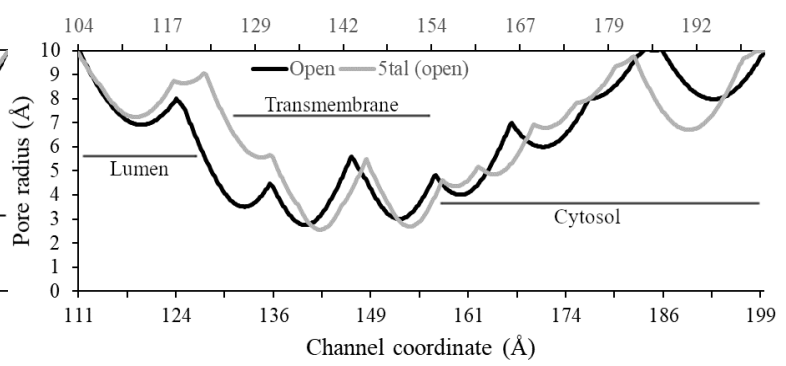


bioRxiv preprint doi: https://doi.org/10.1101/2021.03.09.434632; this version posted March 9, 2021. The copyright holder for this preprint (which was not certified by peer review) is the author/funder. All rights reserved. No reuse allowed without permission.

\section{Figure S1}

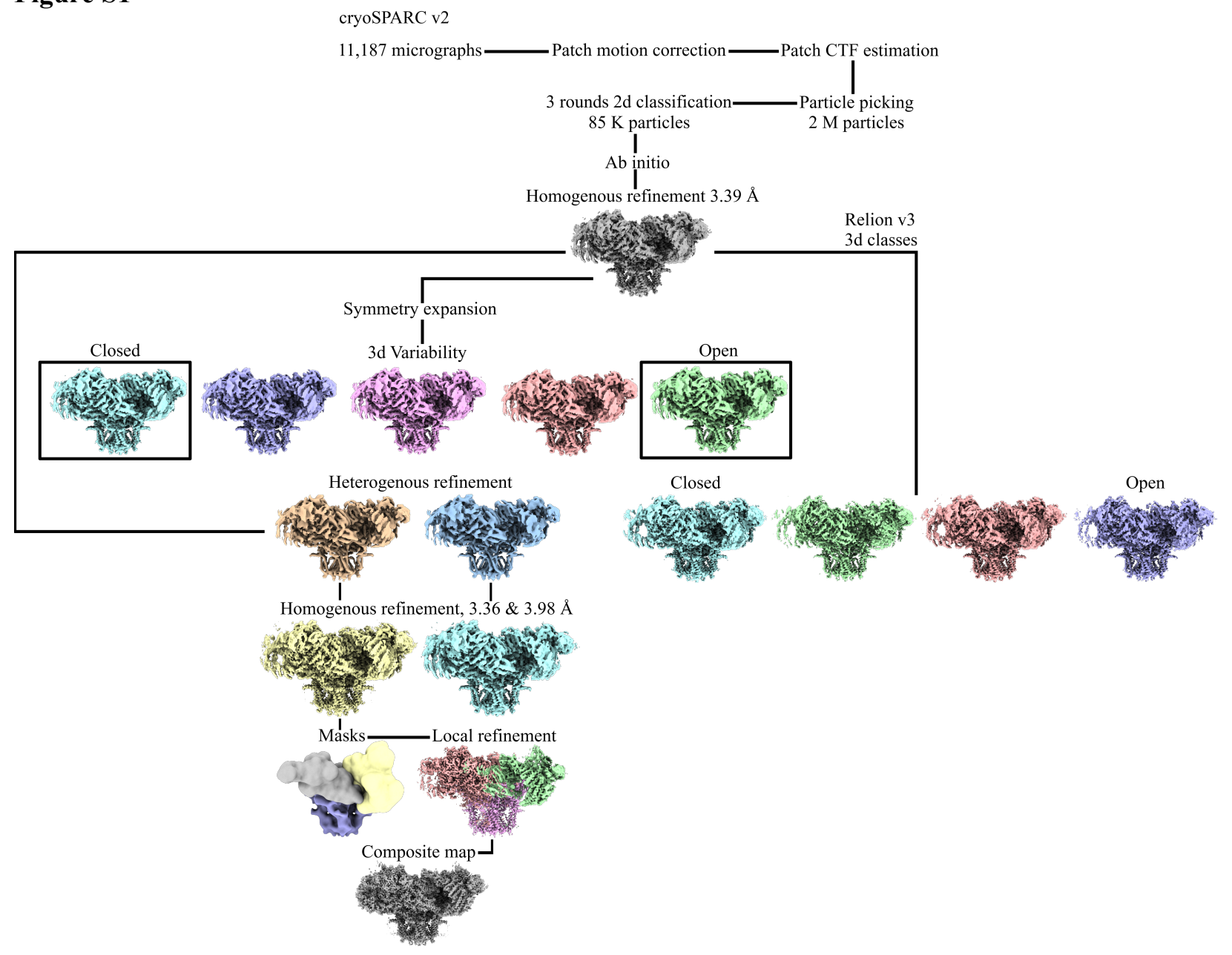


bioRxiv preprint doi: https://doi.org/10.1101/2021.03.09.434632; this version posted March 9, 2021. The copyright holder for this preprint (which was not certified by peer review) is the author/funder. All rights reserved. No reuse allowed without permission.

\section{Figure S2}
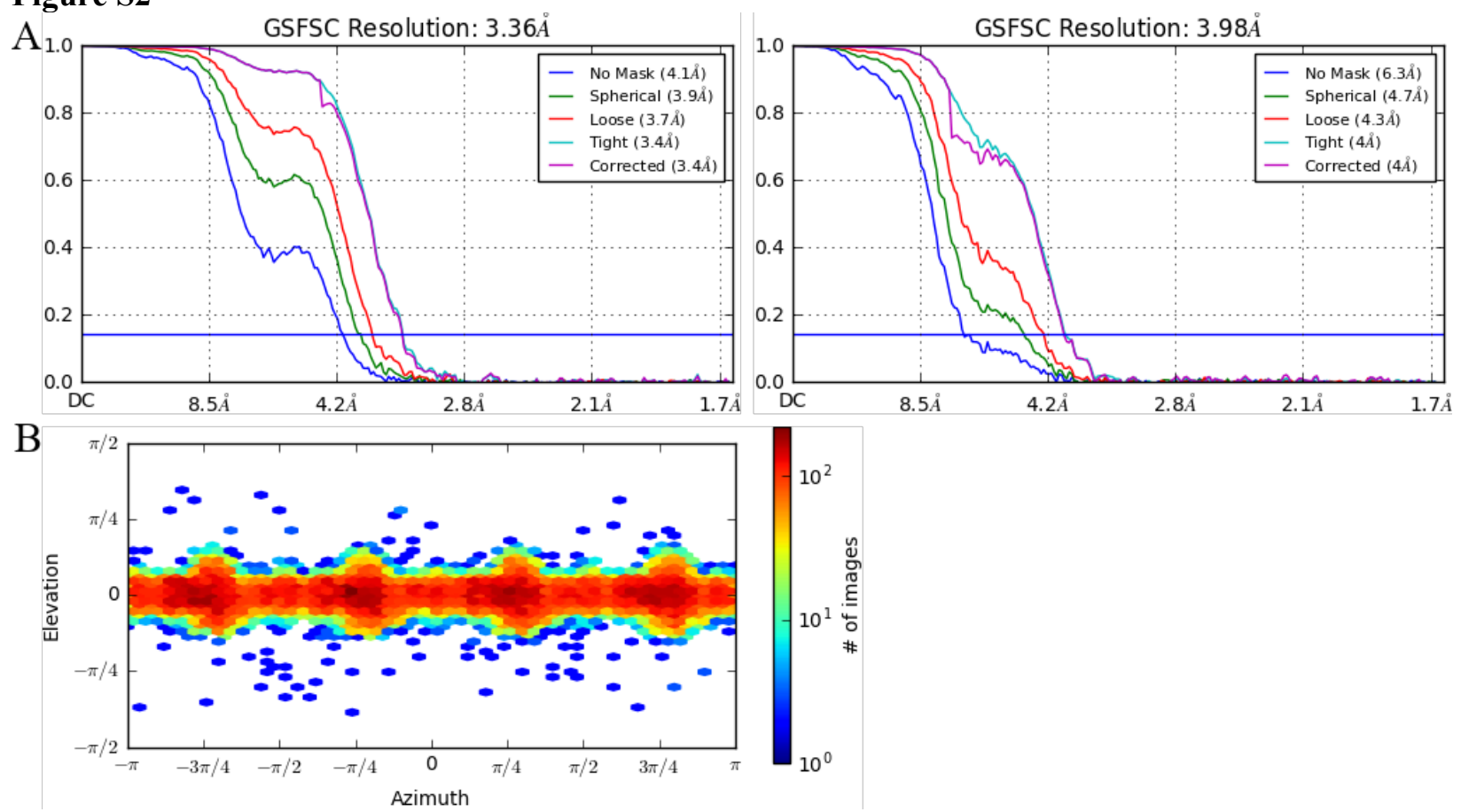


\section{Table S1.}

\begin{tabular}{|c|c|c|}
\hline Data collection & Closed & Open \\
\hline Microscope & \multicolumn{2}{|c|}{ FEI Titan Krios } \\
\hline Detector & \multicolumn{2}{|l|}{ Gatan K3 } \\
\hline Voltage $(\mathrm{kV})$ & \multicolumn{2}{|l|}{300} \\
\hline Magnification & \multicolumn{2}{|l|}{105,000} \\
\hline Exposure $\left(\mathrm{e}^{-} / \AA^{2}\right)$ & \multicolumn{2}{|l|}{58.34} \\
\hline Defocus range $(\mu \mathrm{m})$ & \multicolumn{2}{|l|}{$-1-2$} \\
\hline Pixel size $(\AA)$ & \multicolumn{2}{|l|}{0.831} \\
\hline \multicolumn{3}{|l|}{ Processing } \\
\hline Software & \multicolumn{2}{|l|}{ cryoSPARC } \\
\hline Symmetry & \multicolumn{2}{|l|}{$\mathrm{C} 4$} \\
\hline Initial particles (no.) & \multicolumn{2}{|l|}{85,481} \\
\hline Final particles (no.) & 53,882 & 31,599 \\
\hline Map resolution $(\AA)$ & 3.36 & 3.98 \\
\hline Map resolution range $(\AA)$ & $3.07-3.66^{*}$ & $3.45-4.15^{\ddagger}$ \\
\hline
\end{tabular}

\section{Model Composition}

Peptide chains

12

\begin{tabular}{lc|c} 
Nonhydrogen & 140,672 & 140,428 \\
Protein residues & 17,652 & 17,624 \\
Ligands & 16 &
\end{tabular}

\section{Mean $B$ factors $\left(\AA^{2}\right)$}

\begin{tabular}{ll|l} 
Protein & 83.92 & 97.76 \\
Ligands & 149.86 & 127.35
\end{tabular}

\section{R.m.s. deviations}




\begin{tabular}{ll|l} 
Bond length $(\AA)$ & 0.009 & 0.007 \\
Bond angles $\left(^{\circ}\right)$ & 0.925 & 0.851 \\
Ramachandran & &
\end{tabular}

\section{Ramachandran}

\begin{tabular}{ll|l} 
Favored (\%) & 95.70 & 92.48 \\
Allowed (\%) & 4.25 & 7.50 \\
Disallowed (\%) & 0.05 & 0.02
\end{tabular}

\section{Validation}

\begin{tabular}{ll|l} 
MolProbity score & 2.06 & 2.26 \\
Clashscore & 18.20 & 19.90 \\
Rotamer outliers (\%) & 0.38 & 0.39
\end{tabular}

Map resolution ranges represent the range determined by local refinement in cryoSPARC using the local masks described in Methods. 


\section{References}

1 Willegems, K. \& Efremov, R. G. Influence of Lipid Mimetics on Gating of Ryanodine Receptor. Structure 26, 1303-1313.e1304, doi:https://doi.org/10.1016/j.str.2018.06.010 (2018).

2 Santulli, G., Lewis, D., des Georges, A., Marks, A. R. \& Frank, J. Ryanodine Receptor Structure and Function in Health and Disease. Subcell Biochem 87, 329-352, doi:10.1007/978-981-10-7757-9_11 (2018).

3 Kushnir, A., Wajsberg, B. \& Marks, A. R. Ryanodine receptor dysfunction in human disorders. Biochim Biophys Acta Mol Cell Res 1865, 1687-1697, doi:10.1016/j.bbamcr.2018.07.011 (2018).

4 Marks, A. R. et al. Molecular cloning and characterization of the ryanodine receptor/junctional channel complex cDNA from skeletal muscle sarcoplasmic reticulum. Proc Natl Acad Sci U S A 86, 8683-8687, doi:10.1073/pnas.86.22.8683 (1989).

5 Furuichi, T. et al. Multiple types of ryanodine receptor/Ca2+ release channels are differentially expressed in rabbit brain. J Neurosci 14, 4794-4805, doi:10.1523/jneurosci.14-08-04794.1994 (1994).

6 Giannini, G., Conti, A., Mammarella, S., Scrobogna, M. \& Sorrentino, V. The ryanodine receptor/calcium channel genes are widely and differentially expressed in murine brain and peripheral tissues. J Cell Biol 128, 893-904, doi:10.1083/jcb.128.5.893 (1995).

7 Lanner, J. T., Georgiou, D. K., Joshi, A. D. \& Hamilton, S. L. Ryanodine Receptors: Structure, Expression, Molecular Details, and Function in Calcium Release. Cold Spring Harbor Perspectives in Biology 2, a003996, doi:10.1101/cshperspect.a003996 (2010).

8 Kushnir, A. \& Marks, A. R. The Ryanodine Receptor in Cardiac Physiology and Disease. Advances in pharmacology (San Diego, Calif.) 59, 1-30, doi:10.1016/S10543589(10)59001-X (2010).

9 Marx, S. O. \& Marks, A. R. Dysfunctional ryanodine receptors in the heart: New insights into complex cardiovascular diseases. Journal of molecular and cellular cardiology 58, 225-231, doi:10.1016/j.yjmcc.2013.03.005 (2013).

10 Fabiato, A. Calcium-induced release of calcium from the cardiac sarcoplasmic reticulum. Am J Physiol 245, C1-14 (1983).

11 Fabiato, A. \& Fabiato, F. Calcium-induced release of calcium from the sarcoplasmic reticulum of skinned cells from adult human, dog, cat, rabbit, rat, and frog hearts and from fetal and new-born rat ventricles. Ann N Y Acad Sci 307, 491-522, doi:10.1111/j.1749-6632.1978.tb41979.x (1978).

12 Marx, S. O., Ondrias, K. \& Marks, A. R. Coupled Gating Between Individual Skeletal Muscle Ca2+ Release Channels (Ryanodine Receptors). Science 281, 818-821, doi:10.1126/science.281.5378.818 (1998).

13 Marx Steven, O. et al. Coupled Gating Between Cardiac Calcium Release Channels (Ryanodine Receptors). Circulation Research 88, 1151-1158, doi:10.1161/hh1101.091268 (2001).

14 Porta, M. et al. Coupled gating of skeletal muscle ryanodine receptors is modulated by Ca2+, Mg2+, and ATP. Am J Physiol Cell Physiol 303, C682-C697, doi:10.1152/ajpcell.00150.2012 (2012). 
15 Zalk, R. \& Marks, A. R. Ca(2+) Release Channels Join the 'Resolution Revolution'. Trends Biochem Sci 42, 543-555, doi:10.1016/j.tibs.2017.04.005 (2017).

16 Bellinger, A. M. et al. Hypernitrosylated ryanodine receptor calcium release channels are leaky in dystrophic muscle. Nat Med 15, 325-330, doi:10.1038/nm.1916 (2009).

17 Marx, S. O. et al. PKA Phosphorylation Dissociates FKBP12.6 from the Calcium Release Channel (Ryanodine Receptor): Defective Regulation in Failing Hearts. Cell 101, 365-376, doi:https://doi.org/10.1016/S0092-8674(00)80847-8 (2000).

18 Dridi, H. et al. Intracellular calcium leak in heart failure and atrial fibrillation: a unifying mechanism and therapeutic target. Nat Rev Cardiol, doi:10.1038/s41569-020-0394-8 (2020).

19 Santulli, G. et al. Calcium release channel RyR2 regulates insulin release and glucose homeostasis. J Clin Invest 125, 4316, doi:10.1172/JCl84937 (2015).

20 Dridi, H. et al. Role of defective calcium regulation in cardiorespiratory dysfunction in Huntington's disease. JCl Insight 5, doi:10.1172/jci.insight.140614 (2020).

21 Suzuki, M., Nagai, Y., Wada, K. \& Koike, T. Calcium leak through ryanodine receptor is involved in neuronal death induced by mutant huntingtin. Biochem Biophys Res Commun 429, 18-23, doi:10.1016/j.bbrc.2012.10.107 (2012).

22 Chen, X. et al. Dantrolene is neuroprotective in Huntington's disease transgenic mouse model. Mol Neurodegener 6, 81, doi:10.1186/1750-1326-6-81 (2011).

23 Bussiere, R. et al. Amyloid beta production is regulated by beta2-adrenergic signalingmediated post-translational modifications of the ryanodine receptor. J Biol Chem 292, 10153-10168, doi:10.1074/jbc.M116.743070 (2017).

24 Lacampagne, A. et al. Post-translational remodeling of ryanodine receptor induces calcium leak leading to Alzheimer's disease-likepathologies and cognitive deficits. Acta Neuropathologica 134, 749-767, doi:10.1007/s00401-017-1733-7 (2017).

25 Zalk, R. et al. Structure of a mammalian ryanodine receptor. Nature 517, 44-49, doi:10.1038/nature13950 (2015).

26 des Georges, A. et al. Structural Basis for Gating and Activation of RyR1. Cell 167, 145157.e117, doi:https://doi.org/10.1016/j.cell.2016.08.075 (2016).

27 Wei, R. et al. Structural insights into $\mathrm{Ca}(2+)$-activated long-range allosteric channel gating of RyR1. Cell Res 26, 977-994, doi:10.1038/cr.2016.99 (2016).

28 Bai, X. C., Yan, Z., Wu, J., Li, Z. \& Yan, N. The Central domain of RyR1 is the transducer for long-range allosteric gating of channel opening. Cell Res 26, 995-1006, doi:10.1038/cr.2016.89 (2016).

29 Chi, X. et al. Molecular basis for allosteric regulation of the type 2 ryanodine receptor channel gating by key modulators. Proc Natl Acad Sci U S A 116, 25575-25582, doi:10.1073/pnas.1914451116 (2019).

30 Bayburt, T. H. \& Sligar, S. G. Membrane protein assembly into Nanodiscs. FEBS Lett 584, 1721-1727, doi:10.1016/j.febslet.2009.10.024 (2010).

31 Efremov, R. G., Gatsogiannis, C. \& Raunser, S. in Methods in Enzymology Vol. 594 (ed Christine Ziegler) 1-30 (Academic Press, 2017).

32 Efremov, R. G., Leitner, A., Aebersold, R. \& Raunser, S. Architecture and conformational switch mechanism of the ryanodine receptor. Nature 517, 39, doi:10.1038/nature13916 https://www.nature.com/articles/nature13916\#supplementary-information (2014). 
33 Leitz, A. J., Bayburt, T. H., Barnakov, A. N., Springer, B. A. \& Sligar, S. G. Functional reconstitution of $\beta 2$-adrenergic receptors utilizing self-assembling Nanodisc technology. BioTechniques 40, 601-612, doi:10.2144/000112169 (2006).

34 Nath, A., Atkins, W. M. \& Sligar, S. G. Applications of Phospholipid Bilayer Nanodiscs in the Study of Membranes and Membrane Proteins. Biochemistry 46, 2059-2069, doi:10.1021/bi602371n (2007).

35 Grethen, A., Oluwole, A. O., Danielczak, B., Vargas, C. \& Keller, S. Thermodynamics of nanodisc formation mediated by styrene/maleic acid (2:1) copolymer. Scientific reports 7, 11517-11517, doi:10.1038/s41598-017-11616-z (2017).

36 Oluwole, A. O. et al. Solubilization of Membrane Proteins into Functional Lipid-Bilayer Nanodiscs Using a Diisobutylene/Maleic Acid Copolymer. Angewandte Chemie (International ed. in English) 56, 1919-1924, doi:10.1002/anie.201610778 (2017).

37 Oluwole, A. O. et al. Formation of Lipid-Bilayer Nanodiscs by Diisobutylene/Maleic Acid (DIBMA) Copolymer. Langmuir 33, 14378-14388, doi:10.1021/acs.langmuir.7b03742 (2017).

38 Swainsbury, D. J. K. et al. The effectiveness of styrene-maleic acid (SMA) copolymers for solubilisation of integral membrane proteins from SMA-accessible and SMA-resistant membranes. Biochimica et biophysica acta. Biomembranes 1859, 2133-2143, doi:10.1016/j.bbamem.2017.07.011 (2017).

39 Tonggu, L. \& Wang, L. Cryo-EM sample preparation method for extremely low concentration liposomes. Ultramicroscopy 208, 112849, doi:https://doi.org/10.1016/j.ultramic.2019.112849 (2020).

40 Wang, L. \& Sigworth, F. J. Structure of the BK potassium channel in a lipid membrane from electron cryomicroscopy. Nature 461, 292-295, doi:10.1038/nature08291 (2009).

41 Franzini-Armstrong, C., Protasi, F. \& Ramesh, V. Comparative ultrastructure of Ca2+ release units in skeletal and cardiac muscle. Ann N Y Acad Sci 853, 20-30, doi:10.1111/j.1749-6632.1998.tb08253.x (1998).

42 Sun, X. H. et al. Molecular architecture of membranes involved in excitation-contraction coupling of cardiac muscle. J Cell Biol 129, 659-671, doi:10.1083/jcb.129.3.659 (1995).

43 Franzini-Armstrong, C. \& Jorgensen, A. O. Structure and development of E-C coupling units in skeletal muscle. Annu Rev Physiol 56, 509-534, doi:10.1146/annurev.ph.56.030194.002453 (1994).

44 Smart, O. S., Goodfellow, J. M. \& Wallace, B. A. The pore dimensions of gramicidin A. Biophysical Journal 65, 2455-2460, doi:https://doi.org/10.1016/S0006-3495(93)81293-1 (1993).

45 Santulli, G., Nakashima, R., Yuan, Q. \& Marks, A. R. Intracellular calcium release channels: an update. J Physiol 595, 3041-3051, doi:10.1113/JP272781 (2017).

46 Flucher, B. E. et al. Triad formation: organization and function of the sarcoplasmic reticulum calcium release channel and triadin in normal and dysgenic muscle in vitro. The Journal of cell biology 123, 1161-1174, doi:10.1083/jcb.123.5.1161 (1993).

47 Inui, M., Saito, A. \& Fleischer, S. Purification of the ryanodine receptor and identity with feet structures of junctional terminal cisternae of sarcoplasmic reticulum from fast skeletal muscle. Journal of Biological Chemistry 262, 1740-1747 (1987). 
48 Wang, S. Q., Stern, M. D., Ríos, E. \& Cheng, H. The quantal nature of Ca2+ sparks and in situ operation of the ryanodine receptor array in cardiac cells. Proceedings of the National Academy of Sciences of the United States of America 101, 3979-3984, doi:10.1073/pnas.0306157101 (2004).

49 Chen, W. \& Kudryashev, M. Structure of RyR1 in native membranes. EMBO reports n/a, e49891, doi:10.15252/embr.201949891.

50 Brillantes, A. B. et al. Stabilization of calcium release channel (ryanodine receptor) function by FK506-binding protein. Cell 77, 513-523 (1994).

51 Bers, D. M., Patton, C. W. \& Nuccitelli, R. A practical guide to the preparation of Ca2+ buffers. Methods Cell Biol 40, 3-29, doi:10.1016/s0091-679x(08)61108-5 (1994).

52 Wagenknecht, T., Grassucci, R. \& Frank, J. Electron microscopy and computer image averaging of ice-embedded large ribosomal subunits from Escherichia coli. Journal of Molecular Biology 199, 137-147, doi:https://doi.org/10.1016/0022-2836(88)90384-1 (1988).

53 Jacques Dubochet, M. A., Jiin-ju Chang, Jean-Claude Homo, Jean Lepault, Alasdair W. McDowall, Patrick Shultz. Cryo-electron microscopy of vitrified specimens. $Q$ Rev Biophys 21, 129-228 (1988).

54 Mastronarde, D. N. SerialEM: A Program for Automated Tilt Series Acquisition on Tecnai Microscopes Using Prediction of Specimen Position. Microscopy and Microanalysis 9, 1182-1183, doi:10.1017/S1431927603445911 (2003).

55 Suloway, C. et al. Automated molecular microscopy: The new Leginon system. Journal of Structural Biology 151, 41-60, doi:https://doi.org/10.1016/j.jsb.2005.03.010 (2005).

56 Punjani, A., Rubinstein, J. L., Fleet, D. J. \& Brubaker, M. A. cryoSPARC: algorithms for rapid unsupervised cryo-EM structure determination. Nature Methods 14, 290, doi:10.1038/nmeth.4169

https://www.nature.com/articles/nmeth.4169\#supplementary-information (2017).

57 Rohou, A. \& Grigorieff, N. CTFFIND4: Fast and accurate defocus estimation from electron micrographs. Journal of Structural Biology 192, 216-221, doi:https://doi.org/10.1016/i.jsb.2015.08.008 (2015).

58 Rubinstein, J. L. \& Brubaker, M. A. Alignment of cryo-EM movies of individual particles by optimization of image translations. Journal of Structural Biology 192, 188-195, doi:https://doi.org/10.1016/j.jsb.2015.08.007 (2015).

59 Stagg, S. M., Noble, A. J., Spilman, M. \& Chapman, M. S. ResLog plots as an empirical metric of the quality of cryo-EM reconstructions. Journal of Structural Biology 185, 418426, doi:https://doi.org/10.1016/j.jsb.2013.12.010 (2014).

60 Tan, Y. Z. et al. Addressing preferred specimen orientation in single-particle cryo-EM through tilting. Nature Methods 14, 793, doi:10.1038/nmeth.4347

https://www.nature.com/articles/nmeth.4347\#supplementary-information (2017).

61 Scheres, S. H. W. RELION: Implementation of a Bayesian approach to cryo-EM structure determination. Journal of Structural Biology 180, 519-530, doi:https://doi.org/10.1016/i.jsb.2012.09.006 (2012).

62 Pettersen, E. F. et al. UCSF Chimera-A visualization system for exploratory research and analysis. Journal of Computational Chemistry 25, 1605-1612, doi:10.1002/jcc.20084 (2004). 
63 Tung, C. C., Lobo, P. A., Kimlicka, L. \& Van Petegem, F. The amino-terminal disease hotspot of ryanodine receptors forms a cytoplasmic vestibule. Nature 468, 585-588, doi:10.1038/nature09471 (2010).

64 Emsley, P. \& Cowtan, K. Coot: model-building tools for molecular graphics. Acta Crystallographica Section D 60, 2126-2132, doi:doi:10.1107/S0907444904019158 (2004).

65 Liebschner, D. et al. Macromolecular structure determination using X-rays, neutrons and electrons: recent developments in Phenix. Acta Crystallographica Section D 75, 861-877, doi:doi:10.1107/S2059798319011471 (2019).

66 Adams, P. D. et al. PHENIX: a comprehensive Python-based system for macromolecular structure solution. Acta crystallographica. Section D, Biological crystallography 66, 213221, doi:10.1107/S0907444909052925 (2010).

67 Goddard, T. D. et al. UCSF ChimeraX: Meeting modern challenges in visualization and analysis. Protein Sci 27, 14-25, doi:10.1002/pro.3235 (2018).

68 The PyMOL Molecular Graphics System v. Version 2.0 (Schrödinger, LLC). 